\title{
Efectos metabólicos en pacientes obesos posoperados de bypass gástrico laparoscópico: 5 años de experiencia en un hospital de tercer nivel
}

\author{
Metabolic changes in obese patients after laparoscopic gastric bypass: five-year \\ experience in a tertiary referral hospital
}

José D. Hernández-Marín*, César A. Marrufo-Patrón y Federico López-Rosales

Hospital Regional de Alta Especialidad de la Península de Yucatán, Mérida, Yucatán, México

\begin{abstract}
Resumen
Introducción: La obesidad se define como una enfermedad caracterizada por una acumulación anormal o excesiva de grasa que puede ser periudicial para la salud. La cirugía bariátrica es un método efectivo y seguro en el tratamiento de la obesidad extrema. Materiales y Métodos: Se realizó una revisión y análisis de 90 expedientes clínicos de pacientes post-operados de bypass gástrico laparoscópico. Resultados: De los 38 pacientes incluidos para el presente análisis, 17 pacientes (43.6\%), contaron con el diagnóstico de diabetes mellitus tipo 2 previo al procedimiento quirúrgico y 21 pacientes (56.4\%), carecieron de este diagnóstico previo al procedimiento quirúrgico. En los pacientes no diabéticos, se encontraron diferencias estadísticamente significativas en los valores de índice de masa corporal en la comparación de medias entre el estado basal y a los 12 meses después de haber realizado el bypass gástrico, sin embargo, la hemoglobina glucosilada y la creatinina no mostraron diferencia significativa. En contraste, el grupo de pacientes diabéticos presentó diferencias estadísticamente significativas en las cifras de índice de masa corporal, hemoglobina glucosilada y ácido úrico. Conclusiones: El bypass gástrico laparoscópico proporciona pérdida de peso y reducción del IMC satisfactoria, en específico, el porcentaje de exceso de IMC perdido, con la mejora simultánea de comorbilidades relacionadas con la obesidad.
\end{abstract}

PALABRAS CLAVE: Obesidad. Diabetes. Bypass gástrico. Efectos metabólicos.

\begin{abstract}
Background: Obesity is defined as a disease characterized by an abnormal or excessive accumulation of adipose tissue that may be hazardous to the health. Bariatric surgery is an effective and safe treatment of morbid obesity. Materials and Methods: A review and analysis of 90 clinical records of postoperative patients with laparoscopic gastric bypass was performed. Results: 38 patients were included in the study; 17 (43.6\%) of which were diagnosed with diabetes mellitus before the procedure and 21 patients (56.4\%) without diabetes mellitus. In non-diabetic patients, it was found statistical differences in the value of body-mass index (BMI). In the group of diabetic patients, statistical differences were found in the value of BMI, glycated hemoglobin and uric acid. Conclusions: Laparoscopic gastric bypass (LRYGB) provides a satisfactory weight loss and $B M I$ reduction, specifically, percent of excess BMI lost with the simultaneous improvement of comorbid disease.
\end{abstract}

KEY WORDS: Obesity. Diabetes. Gastric bypass. Metabolic effects.

\author{
Correspondencia: \\ *Federico López-Rosales \\ Calle 7433 x 20 y 22, Fracc. Altabrisa \\ C.P. 97130 , Mérida, Yuc., México \\ E-mail: flr770611 @yahoo.com.mx
}

Fecha de recepción: 14-03-2018

Fecha de aceptación: 24-04-2018

DOI://dx.doi.org/10.24875/CIRU.M18000052
Cir Cir. 2018;86:338-346

Contents available at PubMed www.cirugiaycirujanos.com 


\section{Introducción}

La obesidad es una enfermedad sistémica, crónica, progresiva y multifactorial, que ha alcanzado proporciones epidémicas en el mundo y constituye un grave problema de salud pública debido a que genera un incremento en la morbilidad asociada y en los costos en salud, así como una disminución de la sobrevida y de la calidad de vida ${ }^{1,2}$.

La American Association of Clinical Endocrinologists la ha definido como «enfermedad crónica basada en adiposidad", término que identifica una enfermedad crónica, alude a una base fisiopatológica y evita el estigma relacionado con los múltiples significados de obesidad $^{3}$.

La obesidad es un factor de riesgo para numerosas enfermedades crónicas degenerativas, entre las cuales las más importantes son la diabetes mellitus, enfermedades cardiovasculares y diversos tipo de cáncer. De acuerdo con la Encuesta Nacional de Salud y Nutrición 2016, la obesidad afecta al 38.6\% de las mujeres y el $27.7 \%$ de los hombres. Así mismo, se observó un aumento en la prevalencia de diabetes con respecto al año 2012, al pasar del 9.2 al $9.4 \%$. En todo el país se encuentra una mayor prevalencia en las mujeres que en los hombres $(10.3 \text { vs. } 8.4 \%)^{4}$.

Se estima que el $90 \%$ de los casos de diabetes mellitus tipo 2 son atribuibles al sobrepeso y la obe$\operatorname{sidad}^{5}$. En pacientes adultos, el sobrepeso y la obesidad son causa de cerca del $80 \%$ de los casos de diabetes mellitus tipo 2, el $35 \%$ de los casos de enfermedad cardiovascular isquémica y el $55 \%$ de los casos de enfermedad hipertensiva ${ }^{6}$.

Debido a su función en la regulación metabólica, el tracto gastrointestinal constituye un objetivo clínico y biológico importante para el manejo de la diabetes $^{7}$. En los últimos años, las indicaciones para la cirugía bariátrica han evolucionado de la pérdida de peso al control metabólico ${ }^{8,9}$. Si bien el control glucémico es el objetivo primario, el objetivo final es reducir las complicaciones a largo plazo, tanto microvasculares (nefropatía diabética, neuropatía y retinopatía) como macrovasculares (accidente cerebrovascular, enfermedad coronaria y enfermedad vascular periférica).

Se define "cirugía metabólica» como el uso de cirugía gastrointestinal para tratar la diabetes mellitus y la obesidad. Actualmente se recomienda para pacientes con obesidad de grado III (índice de masa corporal
[IMC] $\geq 40 \mathrm{~kg} / \mathrm{m}^{2}$ ) sin importar el grado de control glucémico, así como para pacientes con obesidad de grado II (IMC 35-39.9 kg/m²) con inadecuado control glucémico a pesar del tratamiento médico adecuado y de realizar cambios en el estilo de vida.

Christou, et al. ${ }^{10}$ reportaron una tasa de mortalidad del $6.2 \%$ en un estudio en el que se hizo un seguimiento de los pacientes por 5 años después de haber realizado la cirugía bariátrica, y concluyeron que el procedimiento quirúrgico disminuye a largo plazo la morbimortalidad de los pacientes obesos, principalmente el riesgo cardiovascular y la enfermedad circulatoria en un $72 \%$. En contraste con lo anterior, Adams, et al. ${ }^{11}$ reportaron una tasa de mortalidad del $4.1 \%$, y además documentaron que la mortalidad a largo plazo en los pacientes posoperados de bypass gástrico laparoscópico disminuye un $40 \%$ en comparación con el grupo control.

Los primeros reportes de mejoras estadísticamente significativas después de la cirugía bariátrica en el control de las cifras glucémicas en el tratamiento de pacientes con diagnóstico de diabetes mellitus tipo 2 y obesidad mórbida fueron presentados en 1987 por Pories, et al. ${ }^{12}$, y a partir de entonces numerosos estudios han demostrado cambios metabólicos tras la cirugía bariátrica.

En 2004, Sjöström, et al. ${ }^{13}$ demostraron que las incidencias de hipertrigliceridemia, diabetes e hiperuricemia eran marcadamente menores en el grupo manejado con cirugía bariátrica que en el grupo control después de 2 y 10 años. En 2008, Lee, et al. ${ }^{14}$ demostraron que el $89.5 \%$ de los pacientes diabéticos con IMC $<35$ habían regresado a la euglucemia 1 año después del bypass, con una reducción de la hemoglobina glucosilada del 7.3 al $5.6 \%$.

Uno de los estudios publicados hasta el momento representativo de lo antes mencionado es el metaanálisis realizado por Buchwald, et al. ${ }^{15}$, quienes documentaron un mejor control glucémico e incluso la remisión de la diabetes después de la cirugía bariátrica durante 1 a 2 años de seguimiento.

Recientemente, dos estudios aleatorizados han continuado demostrando que la cirugía bariátrica conduce a mejores tasas de control de la glucemia que la terapia farmacológica sola. Mingrone, et al. ${ }^{16}$ realizaron un estudio aleatorizado con 60 pacientes entre 30 y 60 años con un IMC > 35 y diabetes de más de 5 años de evolución, que fueron asignados al azar a recibir tratamiento médico o realizar bypass gástrico 0 derivación biliopancreática. A los 2 años 
reportaron la remisión de la diabetes en el 75\% de los pacientes con bypass gástrico y el $95 \%$ de los pacientes con derivación biliopancreática. A los 5 años, el $37 \%$ de los pacientes con bypass gástrico y el $63 \%$ de los pacientes con derivación biliopancreática mantuvieron la remisión de la diabetes, comparado con ninguno de los pacientes con tratamiento médico.

Schauer, et al. ${ }^{17}$ realizaron un estudio aleatorizado y controlado con 150 pacientes diabéticos en el que compararon manejo médico intensivo con bypass gástrico o manga gástrica. A los 3 años, el objetivo primario (hemoglobina glucosilada $<6 \%$ ) fue alcanzado por el $5 \%$ de los pacientes que recibieron tratamiento médico, comparado con el $38 \%$ de los que fueron sometidos a bypass gástrico y el $24 \%$ de los que fueron sometidos a manga gástrica. A los 5 años, el objetivo primario fue alcanzado por el $5 \%$ de los que recibieron manejo médico, comparado con el $29 \%$ en el grupo de bypass gástrico y el $23 \%$ en el grupo de manga gástrica.

Lo anterior supone que, a medida que aumenta el tiempo tras la cirugía, los beneficios de esta disminuyen. Sin embargo, en un seguimiento a 7 años se reporta que la prevalencia de dislipidemia es menor en los pacientes sometidos a bypass gástrico y banda gástrica ajustable; la prevalencia de diabetes y de hipertensión es menor solo en el grupo de bypass gástrico ${ }^{18}$.

Por otra parte, la obesidad se ha implicado como causa de sobreproducción y pobre depuración renal de urato $^{19}$. Los factores identificados que pueden precipitar un episodio agudo de gota incluyen infección, trauma, cirugía, transgresión dietética, nutrición parenteral total e inicio de terapia para disminuir el ácido úrico ${ }^{20}$.

El Hospital Regional de Alta Especialidad de la Península de Yucatán fue el primer hospital institucional del sureste de México en iniciar un programa multidisciplinario de cirugía de obesidad, en el año 2010.

El objetivo de este estudio es describir y comparar los cambios en las cifras de glucosa, hemoglobina glucosilada, colesterol total, triglicéridos, colesterol ligado a lipoproteínas de alta densidad (HDL), colesterol ligado a lipoproteínas de baja densidad (LDL), creatinina, ácido úrico, peso e IMC en los pacientes posoperados de bypass gástrico laparoscópico previo al procedimiento quirúrgico y a los 12 meses de la cirugía en el Hospital Regional de Alta Especialidad de la Península de Yucatán.

\section{Métodos}

La población en estudio son todos los pacientes que hayan cumplido con criterios para cirugía de obesidad, a quienes se les haya realizado bypass gástrico laparoscópico en Y de Roux en el Hospital Regional de Alta Especialidad de la Península de Yucatán en el periodo comprendido entre los años 2010 y 2015. Se realizó una revisión sistemática de 90 expedientes clínicos, de los cuales solo 39 cumplieron con criterios de selección para participar en el estudio.

Se realizó estadística descriptiva de las variables cuantitativas (edad, glucosa sérica, hemoglobina glucosilada, colesterol total, triglicéridos, colesterol total, colesterol HDL, colesterol LDL, creatinina, urea, peso e IMC) dependiendo de su tipo de distribución. Para determinar el tipo de distribución se utilizó la prueba de normalidad de Shapiro-Wilk por el tamaño de muestra de la población en estudio.

Para las variables cualitativas (sexo, diagnóstico de diabetes mellitus tipo 2 previo a la cirugía, diagnóstico de hipertensión arterial previo a la cirugía y diagnóstico de síndrome de apnea obstructiva del sueño previo a la cirugía) se emplearon frecuencias simples y porcentaje.

Por el tipo de distribución gaussiana que presentaron las variables cuantitativas se utilizó estadística paramétrica. Al asumir normalidad se usó la prueba t de Student para muestras relacionadas; para las variables cualitativas se utilizó la prueba de ji al cuadrado; cuando en alguna de las celdas de la tabla de $2 \times 2$ alguno de los valores esperados fue $<5$ se usó la prueba exacta de Fischer. Se consideró una $p<0.05$ como estadísticamente significativa y se utilizó el paquete estadístico SPSS versión 21.0.

\section{Resultados}

Del total de los 90 pacientes intervenidos quirúrgicamente de bypass gástrico laparoscópico en $Y$ de Roux, la muestra se redujo a 38 pacientes porque se descartaron del análisis $52(57 \%)$ debido a pérdida de seguimiento en el Hospital Regional de Alta Especialidad de la Península de Yucatán.

Como se muestra en la tabla 1 , de los 38 pacientes incluidos para el presente análisis, 17 (43.6\%) contaron con el diagnóstico de diabetes mellitus tipo 2 previo al procedimiento quirúrgico. En ambos grupos, con y sin diagnóstico de diabetes, hubo mayor prevalencia de las mujeres, siendo un $88 \%$ (15 pacientes) con diagnóstico de diabetes y un 76\% (16 pacientes) 
Tabla 1. Características sociodemográficas, de antropometría y metabólicas en el estado basal

\begin{tabular}{lcc}
\hline Variable & $\begin{array}{c}\text { Grupo de pacientes } \\
\text { diabéticos } \\
n=17(43.6 \%)\end{array}$ & $\begin{array}{c}\text { Grupo de } \\
\text { pacientes no } \\
\text { diabéticos } \\
n=21(56.4 \%)\end{array}$ \\
\hline
\end{tabular}

Sexo*

$\begin{array}{lcc}\text { Mujeres } & 15(88.2) & 16(76.2) \\ \text { Hombres } & 2(11.8) & 5(23.8) \\ \text { Edad }^{\dagger}(\text { años }) & 41.1(9.9) & 35.1(7.7) \\ \text { Peso }^{\dagger}(\mathrm{kg}) & 118.4(16.7) & 124(22.5) \\ \text { Talla }^{\dagger}(\mathrm{cm}) & 159.8(7.8) & 160.5(9.6) \\ \text { IMC }^{\dagger}\left(\mathrm{kg} / \mathrm{m}^{2}\right) & 46(6.5) & 47.3(4.9) \\ \text { Glucosa }^{\dagger}(\mathrm{mg} / \mathrm{ml}) & 107(12.3) & 93.4(8.4) \\ \text { Hemoglobina } \mathrm{glucosilada} \\ \text { Colesterol total }\end{array}$

"Los datos se expresan como: frecuencias (porcentajes).

†Los datos se presentan como: media (desviación estándar).

HDL: lipoproteínas de alta densidad; IMC: índice de masa corporal; LDL: lipoproteínas

de baja densidad; SAOS: síndrome de apnea obstructiva del sueño

sin diagnóstico de diabetes. La media de edad fue de 41 años en el grupo de los pacientes diabéticos, en comparación con 35 años en el grupo de los no diabéticos. La media registrada para el peso en el grupo de pacientes diabéticos fue de $118.4 \mathrm{~kg}$, con una desviación estándar de $16.7 \mathrm{~kg}$, mientras que en el grupo de pacientes no diabéticos la media registrada para el peso fue de $124 \mathrm{~kg}$ con una desviación estándar de $22.5 \mathrm{~kg}$. Por otra parte, en cuanto a la talla presentada en ambos grupos, en los pacientes diabéticos la media fue de $1.59 \mathrm{~m}$ con una desviación estándar de $7.8 \mathrm{~cm}$, mientras que en los pacientes no diabéticos fue de $1.60 \mathrm{~m}$ con una desviación estándar de $9.6 \mathrm{~cm}$.
Derivado del peso y la talla, la media del IMC en el grupo de pacientes diabéticos fue de $46 \mathrm{~kg} / \mathrm{m}^{2}$, con una desviación estándar de $6.5 \mathrm{~kg}$, mientras que en el grupo de pacientes no diabéticos el IMC presentó una media de $47.3 \mathrm{~kg} / \mathrm{m}^{2}$ con una desviación estándar de $8.4 \mathrm{~kg}$.

En relación con los productos metabólicos, como era de esperar, todos los valores registrados fueron menores en los pacientes no diabéticos, comenzando por la glucosa sérica, con una media de $93.4 \mathrm{mg} / \mathrm{dl}$ y una desviación estándar de $8.4 \mathrm{mg}$, y en concordancia a ese valor, la hemoglobina glucosilada presentó una media de 4.9 con una desviación estándar de 0.5 .

Respecto al perfil lipídico, el colesterol fue el que presentó mayor diferencia de medias entre ambos grupos, con una media de $198 \mathrm{mg} / \mathrm{dl}$ y una desviación estándar de $42 \mathrm{mg}$ en el grupo de pacientes diabéticos, mientras que en el grupo de pacientes no diabéticos presentó una media de $193 \mathrm{mg} / \mathrm{dl}$ y una desviación estándar de $34.7 \mathrm{mg}$. El producto lipídico que presentó menor diferencia de medias entre ambos grupos fue el colesterol HDL, que en el grupo de pacientes diabéticos presentó una media de $44.5 \mathrm{mg} /$ dl y una desviación estándar de $9.8 \mathrm{mg}$, mientras que en el grupo de pacientes no diabéticos presentó una media de $41.7 \mathrm{mg} / \mathrm{dl} \mathrm{y}$ una desviación estándar de $8.9 \mathrm{mg}$.

En los productos metabólicos de la función renal, tanto para la creatinina como para el ácido úrico la diferencia de medias en ambos grupos fue de 0.1.

Finalmente, se observó que el grupo con mayor prevalencia de hipertensión arterial sistémica y síndrome de apnea obstructiva del sueño fue el de los pacientes no diabéticos.

Una vez realizado el bypass gástrico en la población en estudio se registraron nuevamente los valores de antropometría y de estado metabólico de los pacientes en ambos grupos. Se llevó a cabo la prueba t para muestras relacionadas. La prueba de Levene en todas las variables resultó ser no significativa, por lo que se asumió la homogeneidad de varianzas, como se muestra a continuación.

En la tabla 2 puede verse que se encontraron diferencias estadísticamente significativas en todos los valores de antropometría, pero la hemoglobina glucosilada y la creatinina no mostraron diferencia significativa en la comparación de medias entre el estado basal y a los 12 meses de haber realizado el bypass gástrico. Estos hallazgos permiten corroborar el 
Tabla 2. Comparación de los productos metabólicos y de antropometría en el estado basal y a los 12 meses de la cirugía en el grupo de pacientes no diabéticos

\begin{tabular}{|c|c|c|c|c|}
\hline Variable & $\begin{array}{l}\text { Estado } \\
\text { basal }\end{array}$ & 12 meses & $p$ & IC \\
\hline Peso* $(k g)$ & $124(22.5)$ & $80.2(16.3)$ & 0.000 & $33.58-46.41$ \\
\hline $\mathrm{IMC}^{\star}\left(\mathrm{kg} / \mathrm{m}^{2}\right)$ & $47.3(4.9)$ & $31.4(5.5)$ & 0.000 & $13.51-17.63$ \\
\hline $\operatorname{Glucosa}^{*}(\mathrm{mg} / \mathrm{dl})$ & $93.4(8.4)$ & $83.1(7.2)$ & 0.001 & $5.00-14.90$ \\
\hline $\begin{array}{l}\text { Hemoglobina } \\
\text { glucosilada* (\%) }\end{array}$ & $4.9(0.5)$ & $5.1(0.6)$ & 0.283 & $0.21-0.68$ \\
\hline $\begin{array}{l}\text { Colesterol total }{ }^{*} \\
(\mathrm{mg} / \mathrm{dl})\end{array}$ & $193(34.7)$ & $156.7(20.5)$ & 0.000 & $21.76-51.68$ \\
\hline $\begin{array}{l}\text { Colesterol HDL* } \\
(\mathrm{mg} / \mathrm{dl})\end{array}$ & $41.7(8.9)$ & $58.6(9)$ & 0.000 & $11.51-25.05$ \\
\hline Ácido úrico* (mg/dl) & $5.2(1.2)$ & $4(1.2)$ & 0.001 & $0.65-1.93$ \\
\hline $\begin{array}{l}\text { Colesterol LDL* } \\
(\mathrm{mg} / \mathrm{dl})\end{array}$ & $127.1(27.1)$ & $88.6(19.6)$ & 0.000 & $21.76-51.68$ \\
\hline Triglicéridos* (mg/dl) & $166(77.4)$ & 74 (19.9) & 0.000 & $54.29-125.46$ \\
\hline Creatinina* (mg/dl) & $0.6(0.2)$ & $0.6(0.1)$ & 0.537 & $-0.11-0.06$ \\
\hline
\end{tabular}

control metabólico basal, así como la función renal de los pacientes en este grupo.

En contraste, en el grupo de pacientes diabéticos el único producto metabólico que no presentó significancia estadística en la comparación de medias entre el estado basal y a los 12 meses de haber realizado el bypass gástrico fue la creatinina sérica. Esto es de suma importancia, ya que con la significancia estadística resultante se describe un cambio metabólico importante en los pacientes en estudio, cuyo impacto primario comprometería la función renal existiendo una posible descompensación metabólica con la enfermedad de base diagnosticada en los pacientes; sin embargo, la función renal permaneció sin cambio significativo. Como se muestra en la tabla 3 , existe incluso una diferencia en decremento de 0.1 en la media de creatinina sérica a los 12 meses de seguimiento.

Al analizar el resto de los productos metabólicos y las características antropométricas en los pacientes diabéticos, observamos que tanto el IMC como la hemoglobina glucosilada y el ácido úrico, además de tener una diferencia de medias entre el estado basal y a los 12 meses de seguimiento con significancia estadística, presentan intervalos de confianza que son bastante precisos para destacar la importancia de la
Tabla 3. Comparación de los productos metabólicos y de antropometría en el estado basal y a los 12 meses de la cirugía en el grupo de pacientes diabéticos

\begin{tabular}{lcccc}
\hline Variable & $\begin{array}{c}\text { Estado } \\
\text { basal }\end{array}$ & 12 meses & p & IC \\
\hline Peso* $^{*}(\mathrm{~kg})$ & $118.4(16.7)$ & $79.6(12)$ & 0.000 & $34.04-45.96$ \\
$\mathrm{IMC}^{*}\left(\mathrm{~kg} / \mathrm{m}^{2}\right)$ & $46(6.5)$ & $30.3(4.3)$ & 0.000 & $13.24-18.50$ \\
Glucosa* $(\mathrm{mg} / \mathrm{dl})$ & $107(12.3)$ & $81.4(7)$ & 0.000 & $19.18-31.87$ \\
$\begin{array}{l}\text { Hemoglobina } \\
\text { glucosilada*}(\%)\end{array}$ & $6(0.6)$ & $5(0.6)$ & 0.000 & $0.57-1.46$ \\
$\begin{array}{l}\text { Colesterol total* } \\
\text { (mg/dl) }\end{array}$ & $198(42)$ & $171.7(31.2)$ & 0.016 & $5.55-47.03$ \\
$\begin{array}{l}\text { Colesterol HDL* } \\
\text { (mg/dl) }\end{array}$ & $44.5(9.8)$ & $60.3(15.2)$ & 0.001 & $8.41-24.25$ \\
Ácido úrico* (mg/dl) & $5.3(1.0)$ & $3.9(0.9)$ & 0.000 & $0.80-2.10$ \\
$\begin{array}{l}\text { Colesterol LDL* } \\
\text { (mg/dl) }\end{array}$ & $128.9(31)$ & $95.9(25)$ & 0.003 & $12.77-49.36$ \\
\hline
\end{tabular}

Triglicéridos* (mg/dl) 168.3 (71.4) 86.9 (35.5) $0.000 \quad 50.04-112.66$ \begin{tabular}{lllll} 
Creatinina $^{*}(\mathrm{mg} / \mathrm{dl})$ & $0.7(0.1)$ & $0.6(0.1)$ & 0.102 & $-0.01-0.14$ \\
\hline
\end{tabular}

*Los datos se presentan como: media (desviación estándar). IC: intervalo de confianza; HDL: lipoproteínas de alta densidad; IMC: índice de masa corporal; LDL: lipoproteínas de baja densidad.

cirugía bariátrica como medida terapéutica efectiva para la disminución de estos productos metabólicos a los 12 meses de haber realizado el bypass gástrico, y que esta disminución no se atribuyó al azar.

La significancia estadística hallada en ambos grupos es de gran relevancia clínica, ya que se comprueba la eficacia de la cirugía bariátrica como medida terapéutica no solo para la disminución de peso, sino también para el control metabólico de los pacientes, tanto diabéticos como no diabéticos, salvaguardando la estabilidad en la función renal y sin una descompensación metabólica, como podría esperarse en una población tan sensible como son los pacientes con diagnóstico de diabetes mellitus tipo 2. Sin embargo, cabe destacar que los pacientes diabéticos que se consideraron para participar en este estudio contaban con un adecuado control metabólico en el estado basal, como se describe al inicio de los resultados y en la tabla 1.

Es importante destacar una diferencia fundamental entre ambos grupos de estudio. La hemoglobina glucosilada en los pacientes diabéticos presentó una disminución con significancia estadística y un intervalo de confianza estrecho, lo que da respaldo a la aseveración arriba mencionada. La cirugía bariátrica es una medida terapéutica eficaz para la remisión de 
la diabetes, definida como el regreso a cifras normales de glucosa (hemoglobina glucosilada $<6.5 \% 0$ glucosa en ayuno $<100 \mathrm{mg} / \mathrm{dl}$ ) de más 1 año de duración sin terapia farmacológica ${ }^{21}$. En los resultados obtenidos, la glucosa, al igual que la hemoglobina glucosilada, presentó una disminución estadísticamente significativa, ya que la media de la glucosa a los 12 meses de seguimiento fue de $81.4 \mathrm{mg} / \mathrm{dl}$, lo que enriquece la definición de remisión de la diabetes incluso con la desviación estándar presentada (7 mg/dl).

Tal como se muestra en la tabla 4 , al comparar ambos grupos a los 12 meses de seguimiento se contemplaron tres variables de interés: el porcentaje de exceso de IMC perdido, las complicaciones presentadas por los pacientes debidas al procedimiento quirúrgico, y el manejo de dichas complicaciones.

El porcentaje de exceso de IMC perdido ha sido reconocido como el método para comparar diversos tratamientos de la obesidad. Desde su publicación en el año 2007 por Deitel, et al. ${ }^{22}$ se ha adoptado como la medida de resumen estándar para los estudios cuyo objetivo final sea la comparación de pérdida de peso después de llevar a cabo una intervención terapéutica en una población.

En la actualidad existen dos medidas de resumen objetivas para definir la pérdida de peso en los pacientes después de una intervención terapéutica, ya sea farmacológica o quirúrgica: el porcentaje de exceso de peso perdido y el porcentaje de exceso de IMC perdido. En este trabajo se utilizó como medida de resumen el porcentaje de exceso de IMC perdido, ya que cuenta con mayor objetividad y precisión en la pérdida de peso con significancia clínica para los fines particulares del presente estudio. El peso expresado en kilogramos se ha propuesto y utilizado en varios estudios clínicos con la finalidad de comparar principalmente el efecto terapéutico de los fármacos; sin embargo, cuando se reporta una pérdida de $8 \mathrm{~kg}$ en un paciente con diagnó Pstico de obesidad mórbida, en general esta meta no es significativa en la comparación de peso antes y después de una cirugía bariátrica. Además, expresar el peso en kilogramos por sí mismo no está relacionado con la altura, la cual varía considerablemente en las diversas regiones de un mismo país, como es el caso específico de México. Por ejemplo, el peso saludable de un sujeto con talla alta será de mayor relevancia clínica en comparación con el mismo peso en un sujeto de talla baja. Por lo anterior, consideramos de mayor utilidad la comparación de la
Tabla 4. Comparación del porcentaje de exceso de IMC perdido, complicaciones posquirúrgicas y manejo entre el grupo de pacientes diabéticos y el grupo de pacientes no diabéticos

\begin{tabular}{|c|c|c|c|c|}
\hline & $\begin{array}{l}\text { Grupo de } \\
\text { pacientes } \\
\text { diabéticos }\end{array}$ & $\begin{array}{l}\text { Grupo de } \\
\text { pacientes no } \\
\text { diabéticos }\end{array}$ & $p$ & IC \\
\hline$\% \mathrm{EBL}$ & $76.7(14.5)$ & $72.9(23.7)$ & 0.580 & $10.12,17.6$ \\
\hline Complicaciones $^{\dagger}$ & $4(23.5)$ & $2(9.6)$ & 0.378 & \\
\hline Fuga & $3(17.6)$ & $0(0)$ & & \\
\hline Estenosis & $1(5.9)$ & $1(4.8)$ & & \\
\hline $\begin{array}{l}\text { Hernia de } \\
\text { Petersen }\end{array}$ & $0(0)$ & $1(4.8)$ & & \\
\hline Manejo $^{\dagger}$ & $4(23.5)$ & $2(9.6)$ & 0.378 & \\
\hline Ayuno y NPT & $3(17.6)$ & $0(0)$ & & \\
\hline $\begin{array}{l}\text { Dilatación } \\
\text { endoscópica }\end{array}$ & $1(5.9)$ & $1(4.8)$ & & \\
\hline Cierre quirúrgico & $0(0)$ & $1(4.8)$ & & \\
\hline
\end{tabular}

pérdida del exceso de IMC por el constructo que representa.

\section{Discusión}

En este estudio observacional-descriptivo se incluyeron 38 pacientes del Hospital Regional de Alta Especialidad de la Península de Yucatán con diagnóstico de obesidad mórbida a quienes se les realizó bypass gástrico laparoscópico, y se compararon las características antropométricas y metabólicas entre el estado basal y a los 12 meses de haber realizado la cirugía. De los 39 pacientes incluidos, el $43.6 \%$ contaron con el diagnóstico de diabetes mellitus tipo 2 previo a la cirugía, mientras que el $56.4 \%$ carecieron de este diagnóstico; esto supuso una proporción de 1.24:1, pero para fines clínicos podemos puntualizar una relación de 5:4.

Los resultados presentados son concordantes con la literatura médica en relación con las concentraciones séricas elevadas de los factores de riesgo cardiovascular en los pacientes obesos (glucosa, colesterol, triglicéridos, colesterol LDL y ácido úrico), y en cuanto a que estos factores de riesgo disminuyen de forma paralela a la reducción del peso y del IMC una vez realizado el procedimiento quirúrgico. En el presente análisis se observó una disminución de las concentraciones séricas de triglicéridos, colesterol total y colesterol LDL después de la cirugía, mientras que el 
colesterol HDL, con función cardioprotectora, aumentó de forma significativa. La función renal se mantuvo estable, incluso con un decremento no significativo (de 0.1 entre el estado basal y a los 12 meses de seguimiento) de la creatinina sérica en el grupo de pacientes diabéticos. La cirugía bariátrica se asocia con un control metabólico significativo de la glucosa y de la hemoglobina glucosilada en pacientes con diagnóstico de diabetes mellitus tipo 2 y otras enfermedades asociadas a la obesidad, como hipertensión e hiperlipidemia. Lo anterior cuenta con mucha más trascendencia clínica, al conceptualizar a la cirugía bariátrica como un procedimiento quirúrgico que mejora las condiciones metabólicas incluso en pacientes no diabéticos.

En una revisión sistemática y metaanálisis de 136 estudios realizada por Buchwald, et al..$^{15}$ se informó de que el $19.4 \%(n=3769)$ de los pacientes fueron hombres y el $72.6 \%(n=14082)$ fueron mujeres. La media de edad fue de 39 años y la media del IMC para los 16,944 pacientes en el estado basal fue de $46.8 \mathrm{~kg} / \mathrm{m}^{2}$. Lo anterior es concordante con lo hallado en el presente estudio al tener una prevalencia del sexo femenino en ambos grupos por arriba del $75 \%$, pero en cuanto a la edad, las medias son ligeramente discordantes con lo publicado por Buchwald, et al..$^{15}$, ya que en el grupo de pacientes diabéticos la media fue de 41.1 años y en los no diabéticos fue de 35.1 años; sin embargo, no representa una diferencia estadísticamente significativa. El IMC reportado en el estado basal es concordante con lo publicado por dichos autores.

En un estudio realizado por Robert, et al. ${ }^{23}$ se analizó el impacto de la cirugía bariátrica en los factores metabólicos involucrados en la remisión de la diabetes mellitus tipo 2. Se llevó a cabo un seguimiento de los pacientes durante 1 año y se reportó que el $62.8 \%$ presentaron una remisión de la diabetes. Se consideró que los factores predictivos preoperatorios de la remisión de la diabetes fueron el IMC basal $\leq 50 \mathrm{kgm}^{2}$, la hemoglobina glucosilada $\leq 7.1 \%$ y la ausencia de terapia insulínica. Una corta duración de la diabetes y un buen control glucémico preoperatorio aumentan la tasa de remisión de la diabetes mellitus tipo 2 después de 1 año de haber realizado la cirugía.

En 2008, Friedman, et al. ${ }^{24}$ realizaron un estudio prospectivo con 411 pacientes sometidos a bypass gástrico laparoscópico, de los que 21 tenían diagnóstico previo de gota. Siete de los 21 pacientes tuvieron un ataque agudo de gota postoperatorio. Una posible explicación es que a los pacientes posoperados se les indica una ingesta diaria mínima de $60 \mathrm{~g}$ de proteína. En el año 2014, Dalberth, et al. ${ }^{25}$ reportaron un estudio prospectivo en el que 60 pacientes diabéticos se sometieron a una intervención de manga gástrica laparoscópica y fueron seguidos durante 1 año. A las 2 semanas de la cirugía, los valores del ácido úrico aumentaron significativamente, pero disminuyeron a los 3 meses y permanecieron bajos el resto del tiempo de seguimiento.

Lo anterior no es valorable con lo reportado en este estudio, ya que exclusivamente se realizó una segunda medición de las variables en estudio al año de seguimiento, y se desconoce el comportamiento del ácido úrico a las 2 semanas de haber realizado el procedimiento quirúrgico. Sin embargo, se reportó una disminución estadísticamente significativa del ácido úrico en ambos grupos de estudio, así como una funcionalidad renal estable durante ese lapso, con una diferencia en decremento de 0.1 en la media de creatinina sérica en el grupo de pacientes diabéticos a los 12 meses de seguimiento.

Por último, un factor ampliamente considerado en la literatura médica en relación con la cirugía bariátrica, la pérdida de peso y el IMC, así como la remisión de la diabetes mellitus tipo 2 y los productos metabólicos involucrados, es la recuperación de peso y el IMC perdidos en el periodo postoperatorio. Acorde con lo reportado en la literatura, la recuperación de peso comienza entre los 18 y 24 meses después de la cirugía, y un 30 a $64 \%$ de los pacientes experimentan recuperación de peso al transcurrir 2 años desde la cirugía ${ }^{26,27}$.

Los pacientes que se someten a cirugía bariátrica están obligados a cumplir con cambios dietéticos rigurosos con el fin de garantizar la pérdida de peso mantenida y prevenir complicaciones como vómitos y el dumping gástrico ${ }^{28}$.

El cumplimiento de las visitas de seguimiento quirúrgico se ha asociado con hábitos alimentarios mejorados $^{29}$ y mayores grados de actividad física ${ }^{30}$, mientras que la asistencia inadecuada al seguimiento se ha asociado con un menor porcentaje de exceso de peso y de IMC perdidos ${ }^{31,32}$. Es evidente que las conductas y los patrones alimentarios saludables, incluyendo el ejercicio y la asesoría psicológica para el reforzamiento de comportamientos saludables, pueden ayudar a los pacientes a conservar el peso a largo plazo ${ }^{33}$.

Nuestros hallazgos deben ser interpretados dentro del contexto de ciertas limitaciones. En primer lugar, 
el estudio es descriptivo y puramente observacional, lo que impide establecer conclusiones causales sobre los hallazgos reportados. Es conveniente realizar estudios con mayor número de pacientes y con un seguimiento más estrecho durante el primer año postoperatorio, y realizar el seguimiento de los pacientes por al menos 24 meses, periodo crucial para la recuperación del peso y del IMC perdidos. En los pacientes con diagnóstico de diabetes mellitus tipo 2 no se puede establecer la cirugía bariátrica como causa de la mejoría, ya que deben contemplarse el tiempo de diagnóstico y la terapia farmacológica empleada. En caso de contar con estudios que incluyan las características anteriormente mencionadas, los datos podrán serán evaluados usando modelos multivariados con el fin de controlar las variables de metabolismo final a partir de los confusores del estado basal, y con ello establecer conclusiones causales.

\section{Conclusiones}

La obesidad constituye un problema de salud, económico, social y psicológico, que genera mortalidad prematura, incremento en el uso de los servicios de salud, disminución en la calidad de vida, incapacidad y morbilidad crónica; es considerada un pilar fundamental en la incidencia de diabetes mellitus en todo el mundo. El bypass gástrico proporciona una pérdida de peso y una reducción del IMC satisfactorias, en específico en el porcentaje de exceso de IMC perdido, con una mejora simultánea de la comorbilidad relacionada con la obesidad. Los hallazgos del presente estudio son concordantes con lo publicado en la literatura médica; el efecto terapéutico del bypass gástrico laparoscópico se observa más significativamente en los pacientes con un IMC $<50$, lo que implica que el IMC preoperatorio es un fuerte determinante en el resultado final del control metabólico de los pacientes.

El desafío fundamental en el postoperatorio de los pacientes obesos, ya sea que cuenten o no con el diagnóstico de diabetes mellitus tipo 2 , es el mantenimiento de la pérdida de peso $\mathrm{y}$, por ende, el mantenimiento de los valores de los productos metabólicos, tanto de glucosa como del perfil lipídico. Lo anterior radica en el seguimiento estrecho de los pacientes a través de un equipo multidisciplinario, encaminado a la educación del paciente en cuanto a conductas y patrones alimentarios, así como formar el hábito de la actividad física en los pacientes.

\section{Bibliografía}

1. Organización Mundial de la Salud (OMS), Obesidad y sobrepeso. Disponible en: http://www.who.int/es/news-room/fact-sheets/detail/obesity-and-overweight. Consultado Octubre 2017.

2. Hussain SS, Bloom SR. The pharmacological treatment and management of obesity. Postgrad Med. 2011;123:34-44.

3. Mechanick JI, Hurley DL, Garvey WT. Adiposity-based chronic disease as a new diagnostic term: American Association of Clinical Endocrinologists and the American College of Endocrinology position statement. Endocr Pract. 2017;23:372-8.

4. Instituto Nacional de Salud Pública. Encuesta Nacional de Salud y Nutrición de Medio Camino, 2016. Informe final de resultados. Disponible en: http://promocion.salud.gob.mx/dgps/descargas1/doctos_2016/ensanut_mc_2016-310oct.pdf.

5. Dávila-Torres J, González-Izquierdo JJ, Barrera-Cruz A. Panorama de la obesidad en México. Rev Med Inst Mex Seguro Soc. 2015; 53:240-9.

6. Banegas JR, López-García E, Gutiérrez-Fisac JL, Guallar-Castillón P, Rodríguez-Artalejo F. A simple estimate of mortality attributable to excess weight in the European Union. Eur J Clin Nutr. 2003;57:201-8.

7. Rubino F, Nathan DM, Eckel RH, Schauer PR, Alberti KG, Zimmet PF, et al. Metabolic surgery in the treatment algorithm for type 2 diabetes: $a$ joint statement by International Diabetes Organizations. Surg Obes Relat Dis. 2016;12:1144-62.

8. Rubino F, Gagner M, Gentileschi P, Kini S, Fukuyama S, Fenj J, et al. The early effect of the Roux-en-Y gastric bypass on hormones involved in body weight regulation and glucose metabolism. Ann Surg. 2004;240:236-42.

9. Schauer PR, Burguera B, Ikramuddin S, Cottam D, Gourash W, Hamad G, et al. Effect of laparoscopic Roux-en-Y gastric bypass on type 2 diabetes mellitus. Ann Surg. 2003;238:467-84, discussion 484-5.

10. Christou NV, Sampalis JS, Liberman M, Look D, Auger S, McLean AP, et al. Surgery decreases long-term mortality, morbidity and health care use in morbidly obese patients. Ann Surg. 2004;240:416-23.

11. Adams TD, Gress RR, Smith SC, Halverson RC, Simper SC, Rosamond WD, et al. Long-term mortality after gastric bypass surgery. $\mathrm{N}$ Engl J Med. 2007;357:753-61.

12. Pories WJ, Caro JF, Flickinger EG, Meelheim HD, Swanson MS. The control of diabetes mellitus (NIDDM) in the morbidly obese with the Greenville Gastric Bypass. Ann Surg. 1987;206:316-23.

13. Sjöström L, Lindroos AK, Peltonen M, Torgerson J, Bouchard C, Carlsson B, et al. Lifestyle, diabetes, and cardiovascular risk factors 10 years after bariatric surgery. N Engl J Med 2004;351:2683-93.

14. Lee WJ, Wang W, Lee YC, Huang MT, Ser KH, Chen JC. Effect of laparoscopic mini-gastric bypass for type 2 diabetes mellitus: comparison of BMI $>35$ and $<35 \mathrm{~kg} / \mathrm{m}^{2}$. J Gastrointest Surg. 2008;12:945-52.

15. Buchwald $H$, Estok R, Fahrbach K, Banel D, Jensen MD, Poires WJ, et al. Weight and type 2 diabetes after bariatric surgery: systematic review and meta-analysis. Am J Med. 2009;122:248-56.

16. Mingrone G, Panunzi S, De Gaetano A, Guidone C, laconelli A, Nanni G, et al. Bariatric-metabolic surgery versus conventional medical treatment in obese patients with type 2 diabetes: 5 year follow-up of an open-label, single-centre, randomized controlled trial. Lancet. 2015;386:964-73.

17. Schauer PR, Bhatt DL, Kirwan JP, Wolski K, Aminian A, Brethauer SA et al. Bariatric surgery versus intensive medical therapy for diabetes 5-year outcomes. N Engl J Med. 2017;376:641-51.

18. Courcoulas AP, King WC, Belle SH, Berk P, Flum DR, García L, et al. Seven-year trajectories and health outcomes in the Longitudinal Assessment of Bariatric Surgery (LABS) study. JAMA Surg. 2018;153:427-34.

19. Choi HK, Mount DB, Reginato AM. Pathogenesis of gout. Ann Intern Med. 2005;143:499-516.

20. McLean L. The pathogenesis of gout. En: Hochberg MC, editor. Reumathology. $3^{\text {rd }}$ ed. Edinburgh: Mosby; 2003. p. 211-256

21. Buse JB, Caprio S, Cefalu WT, et al. How do we define cure of diabetes? Diabetes Care 2009; 32: 2133-2135.

22. Deitel M, Gawdat K, Melissas J. Reporting weight loss 2007. Obes Surg. 2007;17:565-8.

23. Robert M, Ferrand-Gaillard C, Disse E, Espalieu P, Simon C, Laville M, et al. Predictive factors of type 2 diabetes remission 1 year after bariatric surgery: impact of surgical techniques. Obes Surg. 2013;23:770-5.

24. Friedman JE, Dallal RM, Lord JL. Gouty attacks occur frequently in postoperative gastric bypass patients. Surg Obes Relat Dis. 2008;4:11-5.

25. Dalberth N, Chen P, White M, Gamble GD, Barrat-Boyes C, Gow PJ, et al. Impact of bariatric surgery on serum urate targets in people with 
Cirugía y Cirujanos. 2018;86

morbid obesity and diabetes: a prospective longitudinal study. Ann Rheum Dis. 2014;73:797-802.

26. Hsu LK, Benotti PN, Dwyer J, Roberts SB, Saltzman E, Shikora S, et al. Nonsurgical factors that influence the outcome of bariatric surgery: a review. Psychosom Med. 1998:60:338-46.

27. Magro DO, Geloneze B, Delfini R, Pareja BC, Callejas F, Pareja JC. Long-term weight regain after gastric bypass: a 5-year prospective study. Obes Surg. 2008;18:648-51.

28. Sarwer DB, Dilks RJ, West-Smith L. Dietary intake and eating behavio after bariatric surgery: threats to weight loss maintenance and strategies for success. Surg Obes Relat Dis. 2011;7:644-51.

29. Sarwer DB, Moore RH, Spitzer JC, Wadden TA, Raper SE, Williams NN. A pilot study investigating the efficacy of postoperative dietary counseling to improve outcomes after bariatric surgery. Surg Obes Relat Dis. 2012;8:561-8.

30. Vidal P, Ramón JM, Goday A, Parri A, Crous X, Trillo L, et al. Lack of adherence to follow-up visits after bariatric surgery: reasons and outcome. Obes Surg. 2014;24:179-83.

31. Freire RH, Borges MC, Álvarez-Leite Jl, Toulson Davisson Correia MI. Food quality, physical activity, and nutritional follow-up as determinant of weight regain after Roux-en-Y gastric bypass. Nutrition. 2012;28:53-8.

32. Gould JC, Beverstein G, Reinhardt S, Garren MJ. Impact of routine and long-term follow-up on weight loss after laparoscopic gastric bypass. Surg Obes Relat Dis. 2007;3:627-30.

33. Westenhoefer J, Pudel V. Pleasure from food: importance for food choice and consequences of deliberate restriction. Appetite. 1993;20:246-9. 